\title{
DRAMMATURGIA E SACRE SCRITTURE NELL'ETÀ DEI LUMI
}

\section{FRANCESCO S. MINERVINI}

Lungo tutto il corso del XVIII secolo si assiste alla febbrile ricerca di un modello drammaturgico innovativo; il 'secolo investigante' volgeva cioè la sua peculiare ansia di rinnovamento e il sentimento totalizzante di un'esigenza di razionalizzazione verso una revisione globale e rifondante della scena barocca, afflitta in questa visione da un generale ripiegamento sullo spettacolarismo del gesto come della parola. Si intendeva, quindi, restaurare una drammaturgia regolata, finalmente creata non già dall attore ma per l'attore (Ferrone, 821). In questo senso da un lato l'avvento del libretto sopperiva al cattivo gusto della recitazione all'improvviso (che pure aveva goduto di largo credito e favore del gusto popolare e cortigiano nell'Europa seicentesca) e dall'altro finalmente si intuiva la portata della considerazione del 'teatro come metafora del reale'.

Occasioni e struttura del teatro naturalmente andavano riflettendo lo stato della società settecentesca, ancora cadenzata da una gran messe di ricorrenze liturgiche che ne scandivano (e segnavano profondamente) la successione temporale e le opportunità di incontri culturali. Si pensi che sul finire del secolo lo stesso papa Pio VI dovette intervenire per limitare il numero delle ricorrenze liturgiche (a Roma nel 1770 se ne contavano più di cento): la processione del Santo Chiodo data a Milano o la festività dei Santi Pietro e Paolo il 29 giugno a Roma mostravano la potenzialità della solennità scenica che a queste ricorrenze era tradizionalmente riservata. La città eterna, peraltro, favoriva questi eventi teatrali, basti pensare alla piazza Santi Apostoli o piazza Farnese, veri e propri impianti scenografici all'aperto, immediatamente presenti alla disponibilità dell'organizzazione culturale. Si aggiunga poi che nelle feste di patronato l'occasione per la divulgazione liturgica e la trattazione scritturistica si innervavano nella tensione oppositiva al dilagante giansenismo e in sostegno agli ordini religiosi pesantemente messi in crisi ed offuscati dai nuovi stili di vita in via di affermazione ${ }^{1}$.

Tuttavia, la scena italiana appariva mortificata da un lato dalla mancanza di attori veri e dall'altro dall'assenza di una consapevole progettual-

${ }^{1}$ Si veda a riguardo Orfeo in Arcadia oppure La festa a Roma dal Rinascimento al 1870 . 
ità teatrale in grado di contrastare e finalmente debellare la supremazia dell'ormai troppo abusata Commedia dell'Arte che aveva cancellato (peraltro a vantaggio del melodramma e dell'opera buffa) il lavorio intellettuale intorno a temi e situazioni più confacenti alla nuova realtà politica, sociale e morale del XVIII secolo. I mille difetti del teatro, la scarsezza di forza dei repertori svuotati di ogni pregnanza morale erano già stati messi in evidenza da L.A. Muratori quando nel 1706, trattando Della perfetta poesia italiana, aveva indicato nei repertori vuoti e privi di forza la responsabilità della mancanza in Italia di tragedie che potessero porsi in gara con gli esempi più luminosi della drammaturgie classique francese e che ne ripetessero (eguagliandola o magari superandola) l'elevata dignità. Muratori argomentava le sue tesi focalizzando l'attenzione sulla carenza o addirittura assenza di tensione ideologica nella drammaturgia e nella pratica teatrale italiana; secondo un'indicazione che poi sarà ampiamente condivisa e pugnacemente sostenuta dai teorici di poetica teatrale (Martello, Gravina e Quadrio) si andava definitivamente affermando l'esigenza di trasformare la pratica teatrale da 'puro divertimento a spettacolo di cultura'2. In questo senso opera la diffusione delle traduzioni e delle imitazioni del teatro francese secentesco ${ }^{3}$, di Corneille e di Racine in primo luogo (Ifigenia in Aulide di Racine e Rodoguna di Corneille), testimoniato dalle opere di Gasparo Gozzi, Francesco Albergati Capacelli, Giuseppe Baretti e Melchiorre Cesarotti.

Il maggior merito di Muratori era stato indubbiamente quello di avere compreso e mostrato la necessità di una innovazione e rivisitazione delle teorie tragiche ${ }^{4}$ che finalmente contemplassero, pur nell'adesione alla norma aristotelica ${ }^{5}$, l'apertura alle più moderne elaborazioni come quella di Voltaire o più in là quella di Lessing, soprattutto quando intorno alla metà del secolo si affermò tanto in sede religiosa quanto in campo laico la discus-

${ }^{2}$ Per la trattatistica sui teatri cfr. Marotti, Lo spazio scenico.

${ }^{3}$ Cfr. Ferrari, Le traduzioni italiane del teatro tragico francese, Mangini, "Il teatro tragico francese in Italia"; Meregazzi, Le tragedie di Pierre Corneille, De Angelis, Critiche, traduzioni ed imitazioni del teatro di G. Racine, De Carli, Riflessi francesi nell'opera di Saverio Bettinelli.

${ }^{4}$ Cfr. Bertana, "La tragedia"; Id., "Il teatro tragico italiano del secolo XVIII prima dell'Alfieri"; Binni, "L'aspirazione al teatro tragico"; Doglio, Il teatro tragico italiano; Galletti, "Le teorie drammatiche e la tragedia nel secolo XVIII prima dell'Alfieri"; Mattioda, Le teorie della tragedia nel Settecento; Brizzi, "Caratteri ed evoluzione del teatro di collegio italiano"; Carlson, Teorie del teatro; Luciani, Le passioni e gli affelii.

5 Ariani, "L'ossessione delle 'regole' e il disordine degli 'affetti". 
sione intorno ai concetti di compassione e terrore, orrore e meraviglia, passione amorosa. Alle teorie tragiche si accompagnava inoltre il tentativo di giustificare o quanto meno spiegare le motivazioni della tragedia a lieto fine che si andava via via affermando, riscuotendo soprattutto il favore degli ambienti più attenti alle indicazioni morali della religione cristiana ${ }^{6}$.

Nel 1716 Gian Vincenzo Gravina (Della tragedia) poneva la questione della superiorità del modello tragico greco su quello latino e della irrinunciabilità delle unirà aristoteliche, accordando il suo favore alla tragedia di argomento storico, la quale poteva tra l'altro assolvere una funzione spiccatamente politica. Una posizione questa naturalmente non condivisibile dagli autori guidati da una più convinta fede cristiana o ispirati da quella morale. Nel 1729 infatti Annibale Marchese pubblicava le sue Tragedie cristiane, una raccolta di drammi sacri nel pieno rispetto delle unità aristoteliche e nel 1732 Giulio Cesare Becelli nel trattare Della novella poesia argomentava sulla necessità di fondere il nocciolo tematico della tragedia cristiana con la norma strutturale aristotelica. Queste posizioni collidono naturalmente con quelle dei 'modernisti' come Gorini Corio, Gian Rinaldo Carli', Pier Jacopo Martello ${ }^{8}$, Antonio Conti e Scipione Maffei, convinti invece della ineguagliabile superiorità del modello tragico francese.

Se le due correnti paiono almeno esteriormente non trovare punti di contatto, l'entusiasmo per la virtù sembra invece avvicinarle; l'adesione illuministica al sensismo e la spiccata attenzione ai valori etici e alla moralità (e moralizzazione) teatrale della società finiscono col convergere intorno alle potenzialità educative della pratica teatrale. Tra i primi a cogliere tali possibilità pedagogiche furono naturalmente i Gesuiti, tenaci amministratori di collegi e centri di formazione di vario livello, aperti tuttavia a raccogliere non soltanto le istanze della religione cristiana ma anche le sollecitazioni etiche della drammaturgia francese secentesca, di cui proposero numerosi adattamenti e traduzioni?

La produzione drammaturgica dei Gesuiti si connota tra il 1770 e il 1773 per un "ritorno pressoché corale ai temi biblici"10, con un'adesione a tematiche spirituali di edificazione morale e di preparazione retorica per gli

${ }^{6}$ Cfr. Sannia Nowé, "Epifanie e metamorfosi”; Minervini, "L’etica aristotelica".

${ }^{7}$ Carli, Dellindole del teatro tragico antico e moderno.

${ }^{8} \mathrm{Cfr}$. Martello, Della tragedia antica e moderna.

${ }^{9} \mathrm{Cfr}$. Simioni, Per la storia del teatro gesuitico; Ferrari, "Appunti sul teatro tragico dei Gesuiti"; Scaduto, "Il teatro gesuitico"; I Gesuiti e i primordi del teatro barocco in Europa.

${ }^{10}$ See Five Comedies from the Italian Renaissance. 166, n. 139 
educandi a calcare la scena politica futura; prova ne siano le tragedie di Anton Maria Ambrogi (Morte di Gionata Maccabeo e Gioas, riadattamenti di tragedie di Voltaire) e di Simon Maria Poggi che 'copia' l'Idomeneo di Crebillon, pur depurandolo dall'elemento sentimentale, e quelle di Giuseppe Carpani (Sannacherib), di Davide Scotti (David).

La scelta della tragedia a lieto fine agiva proprio nella direzione della conciliazione tra il modello teatrale francese e le istanze moralistiche religiose; ma d'altro canto essa serviva anche ad allontanare quel 'fatalismo' della tragedia greca che era divenuto simbolo e sostegno ideologico per un dilagante giansenismo che sposava le tesi della redenzione e del libero arbitrio.

I seminaria nobilium, sedi degli educandati gesuitici, divenivano luoghi di elezione per la produzione e spesso anche sperimentazione teatrale illuministica italiana: si pensi al San Luigi di Bologna o a quello dei Nobili di Parma, dove operarono il genovese Giovanni Granelli (1703-1770), il teorizzatore della tragedia religiosa, e poco più tardi il maggiore tragico della Compagnia, Saverio Betrinelli. Sin dalle sue prime opere, Granelli si era mostrato attento interprete dell'esigenza del rinnovamento teatrale e della tragedia religiosa che intendeva ravvivare per il tramite della scrittura versificata, accompagnandola con il genere delle 'lezioni sacre di argomento scritturistico' come momento preparatorio alla ponderosità interpretativa del tema sulla scena teatrale. Predicatore di corte a Vienna presso Maria Teresa d'Austria, Granelli compose nel 1761 Seila figlia di Jefte, tragedia d'argomento israelita; nell'attività presso la corte imperiale, dunque, si andavano fondendo l'adesione a questioni politico-mecenatistiche con l'imprescindibile austerità della tematica scritturistica: in quest'ottica forse si deve anche spiegare la convinzione di Granelli che "le virtù di una tragedia vi sono solo se c'è il favore del pubblico" (Prefazione a Seila, viII). Parole nelle quali vibra il desiderio di cogliere il favore della committenza e ad un tempo anche quello del pubblico, nella speranzosa convinzione umanistica di giovare tanto alla prima nella gestione illuminata del potere, quanto alla edificazione morale dell'uditorio.

Si era ormai concretizzato lo scarto con il passato, la mutazione del gusto cominciava a dare i suoi effetti concreti: ciò che muoveva l'uditorio e gli scrittori di teatro, che pervadeva l'intera società a partire dal Settecento e che si approfondirà nel dibatrito romantico era, dunque, "un sentimento profondo e sublime che scaturisce dalla meditazione sui grandi temi del destino umano, del rapporto fra l'uomo e Dio, l'uomo e il tempo, l'uomo e le forze della natura, o che a quella meditazione sospinge" (Puppo, 51).

La scelta del meraviglioso cristiano necessitava, tuttavia, di essere modificata e plasmata per poter coesistere col sistema drammaturgico classico, 
fortemente intriso di strutture e tematiche decisamente pagane; così come anche la scelta della tematica scritturistica era tuttavia indirizzata al perseguimento di obiettivi pedagogici e pratici di natura laica, sollevando anche in questo caso l'inquisitoria attenzione delle gerarchie ecclesiastiche, chiaramente preoccupate di scongiurare devianti forzature interpretative e rappresentative del testo biblico ${ }^{11}$. Interprete delle ansie rigoristiche religiose, il padre Daniele Concina, pensatore e rigido teorico, si fece portavoce dell'esigenza di un rinnovato rigorismo cattolico ${ }^{12}$, vietando l'introduzione sulle scene dei Collegi delle figure femminili e battendosi per una tragedia aristotelicamente 'regolata' e sorvegliata nella morale. Certamente di fronte a tale intransigenza, la scelta di Granelli di attingere alla storia d'Israele e di intitolare una rappresentazione drammaturgica ad una figura femminile (Seila) doveva naturalmente suscitare preoccupazioni fra i Padri Generali dell'Ordine. Saverio Bettinelli, il maggior drammaturgo della Compagnia, ricorse invece ad un sapiente artifizio retorico; nelle sue tragedie (Gionata figlio di Saul; Demetrio Poliorcete, Serse re di Persia) le donne non appaiono mai direttamente sulla scena, eppure esse sono ugualmente rappresentate, descritte e fatte rivivere attraverso i racconti e nelle parole dei protagonisti maschili: esemplare il caso della madre di Gionata che giunge a suscitare in sollevazione il popolo contro l'intento - dichiarato irremovibile — di Saul di uccidere suo figlio, senza però che tale parte sia sostenuta da un attore sulla scena; e ancora secondo il modello dell'apparizione di Nino nella Semiramis di Voltaire, Amestri moglie di Serse re di Persia da lui stesso assassinata in nome della ragion di Stato continua ad aggirarsi intorno al tiranno afflitto, a lasciare tracce materiali della sua esistenza (un brandello delle vesti, una lettera segreta) come farà anche Stratonica, figlia di Demetrio, innamorata di Cleomene, primogenito del nemico arconte ateniese Timandro. Bettinelli, come si vede, è riuscito nell'intento di mantenere fede alle istanze moralistiche dettate dalla Ratio studiorum e contemporaneamente di salvare tanto la fedeltà al testo (biblico o storico), quanto quella ai bisogni della rappresentazione. È però singolare come i drammaturghi gesuiti ricorrano più di frequente alla storia laica (greca o romana) piuttosto che a quella scritturistica; solo marginale appare infatti a detta dello stesso Bettinelli la prova del Gionata figlio di Saul; nel

11 "Tragoediarum, et Comoediarum, quae non nisi latinas, ac rarissimas esse oportet, argumentum sacrum sit, ac pium, neque quicquam actibus interponantur, quod non latinum sit, et decorum; nec persona ulla muliebris, vel habitus introducantur": Regulae Rectoris n. 13, Ratio studiorum et Institutiones Scholasticae Societatis Jesu.

${ }^{12}$ Concina, De spectaculis, Id., De' teatri moderni contrari alla professione cristiana. 
Discorso del teatro italiano il gesuita ammette i limiti strutturali della sua unica tragedia di argomento biblico, per 'fracchezza di stile' e per una troppo pedissequa imitazione della linea tragica Euripide-Racine, esprimendosi in questi termini:

In quanto a me protesto d'aver tentato seguir queste tracce, ben conoscendomi assai fiacco ne' miei talenti, come il sono in sanità di temperamento. Nel Gionata è molto di morale come nell' Ifigenia d'Euripide $e$ in alcune scene di lui e di Racine. Quest'era pel primo un argomento sacro, non pel secondo, onde questi usò meno della morale che non il primo, ch'io dovea seguire nel Gionata. E perciò presi uno stile più grandioso e poetico sulla scorta de' greci e d'Eschilo principalmente ${ }^{13}$.

Al centro della drammaturgia di collegio opera dunque una forte tensione non solo tematica verso il modello scritturistico che consustanzia la scelta della materia e la cristianizzazione del meraviglioso; ma accanto a ciò è indiscutibile l'attenzione moralistico-pedagogica nei confronti degli educandi considerati nel loro essere "giovani scelti per un grande scopo"14.

La tragedia biblica assume allora contorni sui generis, l'afflato tragico del modello classico greco si stempera via via in una pacata riconciliazione finale, che tende ad uno scioglimento del nodo tragico che muta il volto tetro e cruento, misterioso e punitivo del Fato in quello salvifico e benevolo della grazia della Provvidenza, anche ammettendo l'inserimento di un intreccio risolutivo alternativo.

Nella tenuta ideologica della tragedia si agita, inoltre, il consueto dibattito intorno al verisimile; la notevole produzione di tragedie d'argomento biblico poneva com’è ovvio la questione di un verisimile, quello cristiano, che si innalzasse a competere con lo statuto aristotelico storicamente dominante ${ }^{15}$. Le tragedie scritturistiche di Granelli e Bettinelli mostrano il tentativo programmatico di introduzione sulla scena tragica di un meraviglioso cristiano capace di contemplare al suo interno tanto la statura aristotelica dell'eroe tragico, quanto la natura decisamente meno conflittuale dell'eroe (o martire) cristiano. Il conflitto tragico delle tragedie bibliche è naturalmente di gran lunga meno profondo dei corrispettivi d'ispirazione classica: i protagonisti della tragedia antica e di quella d'argo-

${ }^{13}$ Bettinelli, "Discorso sopra il teatro italiano" pp. 1134-1135; anche cfr. Bonora, "Le tragedie e la poetica del tragico di Saverio Bettinelli".

${ }^{14}$ Cosi Samuele a Gionata nel Gionata, figlio di Saul $(I, 6)$ di Saverio Bettinelli, in Bettinelli, Tomo sesto.

${ }^{15}$ Cfr. Bancheri, Polemiche sulla tragedia sacra, pp. 177-181. 
mento storico greco o romano non sono certamente paragonabili alla quasi irreale drittura morale dei personaggi biblici; in essi vibra un'aura di perfezione che mal si concilia con le richieste istituzionali della tragedia aristotelica. Manca tanto la grandezza della colpa tragica, quanto la bassezza degli eroi 'fallibili' da cui risorgere. Nel primo caso si tratta infatti di colpa o 'errori' (veri sviamenti dalla retta via) di piccola entità, assai spesso inconsapevolmente commessi come nel Gionata di Bettinelli, o ancor più come per l'innocente Manasse di Granelli16. I "giovani scelti per un grande scopo", protagonisti e referenti della drammaturgia gesuitica, sono preventivamente riconosciuti in possesso di un eroismo o eroicità per definizione anti-tragica: essi non possono che mostrare la perfettibilità della loro già perfetta condizione morale attraverso un percorso di redenzione-riconoscimento dell'errore, della colpa tragica, già scritto e provvidenzialmente disposto. Colpa ed errore tragico allora assumono connotati 'esclusivamente' biblici: laddove all'eroe classico (più verosimilmente) era lasciato l'arbitrio di sbagliare e redimersi, originando un nodo tragico il cui scioglimento era tanto più forte e catarticamente efficace quanto maggiore lo sbaglio, all'eroe cristiano è dato soltanto remissivamente interpretare un ruolo già scritto, dogmaticamente immodificabile per sua stessa natura.

Con la produzione gesuitica cui andrà anche accostato per comunione d'intenti il Giovanni di Giscala di Alfonso Varano e la proliferazione della tragedia biblica anche di autori non religiosi, si assiste a un progressivo scarto anche sul campo del verisimile tra il versante cristiano da un lato e quello classico di derivazione storica dall'altro.

Più volte nel Settecento questo problema ha suscitato l'attenzione della critica sin dalla polemica tra Varano e Metastasio, coinvolgendo a vario titolo filosofi o tragediografi. Quel che qui interessa è tuttavia un altro punto: ci si deve interrogare sulle motivazioni che hanno ad esempio Bettinelli, allievo del "Sofocle redivivo" Granelli, a rinunciare alla tragedia scritturistica per dedicarsi ad argomenti della storia greco-persiana (Demetrio e Serse) ed invece un autore di estrazione 'laica' come Alfieri ad assumere il cimento biblico.

La tragediabilità del Saul "come soggetto sacro" giungeva, infatti, in Alfieri nella primavera del 1782 a seguito di quello che egli stesso ebbe a definire un vero invasamento ${ }^{17}$; la scelta del racconto del primo libro di Samuele aveva ovviamente per Bettinelli altre ragioni. Per il gesuita man-

${ }^{16}$ Granelli, Manasse Re Di Giuda. Sedecia Ultimo Re Di Giuda. Dione Siracusano. ${ }^{17}$ Alfieri, Vita IV, 9, pp. 196-197; si veda, inoltre, Parere su Saul, in Alfieri, Parere sulle tragedie e altre prose critiche. 
tovano, il Gionata rappresenta l'esordio tragico, innervato naturalmente nel solco della tradizione gesuitica che egli stesso contribuì a sostenere. La preferenza accordata al tema scritturistico appariva scontata, quasi come per una forma di devozionale osservanza della norma spirituale, retorica, pedagogica e teatrale della Ratio di Sant'Ignazio di Loyola ${ }^{18}$. Il Gionata appare tutto calibrato sulla figura del 'figlio di Saul' come recita il titolo stesso, nel chiaro intento di mostrare le qualità salvifiche in terra come per la beatitudine finale della remissiva e pacata accettazione del volere superiore di Saul, re sulla terra, e ancor più di quello di Dio, il re dei re. La conformità del comportamento di Gionata (inconsapevolmente macchiatosi di una colpa oggettivamente 'minore' come l'aver attinto una stilla di miele durante la battaglia) ad un canone etico riconosciuto come l'unico valido dai committenti, dall'autore e dagli attori-spettatori è l'autorappresentazione di una realtà chiusa e autoreferenziale che si specchia in se stessa. Una realtà che doveva apparire certamente ristretta allo stesso Bettinelli che subito dopo abbandonerà la tematica biblica in favore di quella storica, per potersi più chiaramente e liberamente dedicare alla questione politica e sociale più evidente nel Serse e nel Demetrio e che segue più da vicino la tradizione gesuitica della letteratura sulla Ragion di Stato ${ }^{19}$.

La rigida e dogmatica definizione della storia biblica, l'immodificabilità di una fabula preventivamente accettata, l'impossibilità di plasmare il 'meraviglioso cristiano' (che nel Gionata si concretizza nel 'provvidenziale' sollevamento popolare provocato dalla vigorosa e tenace determinazione della madre del protagonista eponimo) dovevano indubbiamente apparire coercitive sia sul piano tematico sia su quello dell'adeguamento alle strutture normative delle unità aristoteliche e soprattutto carenti nella tensione politica e sociale che gli educandi stessi avrebbero incontrato sulla scena della vita reale.

La quaestio era assai dibattuta e non è qui il caso di ricordare un conflitto di ragioni che dalla Poetica di Aristotele si inasprisce nel Cinquecento e si protrae sino alla nascita del dramma borghese. Certamente è più interessante soffermarsi sui modi di intendere e sviluppare la materia vetero-testamentaria tra i Gesuiti da un lato e un autore 'laico' come 'Alfieri' dall'altro. Resta valido pertanto a mio avviso il discernimento che a proposito diede Attilio Simioni a proposito del gesuita Giovan Battista Roberti (17191786): "Il soggetto biblico, in mano d'un vero tragico, o forse anche del Bettinelli stesso, se le regole ed il fine non gli avessero inceppate spesse volte le mani, si sarebbe prestato, se non ad un intreccio fortemente drammatico,

${ }^{18}$ Cfr. I Gesuiti e la Ratio Studiorum.

${ }^{19}$ Cfr. Ragion di Stato a Teatro. 
certo ad un'azione molto più vivace di colorito affettivo e passionale" (Simioni, 15). Simioni parlava dell'Adonia (1734) di G.B. Roberti, la cui fonte biblica è la medesima del Gionata di Bettinelli e del Saul alfieriano, ovvero "la fatale persecuzione di Dio" contro la casa dei regnanti di Israele (Simioni, 13). Mi preme segnalare, prima di giungere al confronto con l'Alfieri, che nonostante la tenuta tematica tutta incentrata sulla storia biblica, queste opere mostrano un afflato del tutto peculiare ed allotrio rispetto alla loro natura esteriore; voglio dire che pur in un contesto biblico (il più vicino alla tradizione e alla cultura di questi tragediografi) l'attenzione principale pare riservata al tema del potere, del conflitto con l'animo umano e più in generale alla discussione sulla Ragion di Stato che aveva già riscosso in passato l'attenzione di gesuiti come Botero e Traiano Boccalini. Né si può tralasciare che la drammatizzazione del Saul giungeva non soltanto dopo l' invasamento" biblico del 1782, ma soprattutto alla fine di un percorso ideologico e letterario che già lo aveva portato l'Alfieri alla profondità della riflessione sulla Tirannide (1777), alla composizione delle cosiddette 'tragedie della libertà' (Virginia, Timoleone, La congiura dei pazzi, 17771779), alla trattazione Del principe e delle lettere e di seguito del Filippo, concepito già nel 1777 ma terminato insieme col Polinice nel $1783^{20}$.

Se prendiamo in esame anche le opere di Granelli come il Sedecia, ben si comprende tale ipotesi: Sedecia - la cui vicenda è narrata in II, Cronache 36: 11-21 - è la raffigurazione teatrale di una dimensione umana pienamente 'politica' e in tal senso peculiarmente valida a livello pedagogico come 'vita negativa', ovvero come fulgido esempio etico da rifiutare e attraverso cui educare antifrasticamente al bene gli allievi; così appare, secondo categorie di giudizio delle Vite parallele di Plutarco, il giovanissimo (appena ventunenne) re di Gerusalemme, Sedecia, un tempo fedele vassallo di Nabucodonosor, quindi (nel 588 a. C.) passato dalla parte del faraone Hofra, quindi fatalmente espostosi alla vendetta del primo; puntualmente, come in ossequio ad una legge immodificabile della politica, tale vendetta ebbe luogo con la presa di Gerusalemme da parte del sovrano babilonese il quale procedette ad uccidere i figli di Sedecia in sua stessa presenza, accecato e ridotto in vincoli. La drammaticità dell'evento storico-biblico dell'ultimo re di Giuda comprende nel suo intreccio la constatazione della bramosia umana di potere, dell'attaccamento ostinato ad esso, nonché il conflitto tra il volere supremo di Dio e la hybris dell'uomo, la contrapposizione tra il cieco furore del potente e la visione della sofferenza dei figli, biblicamente ricettori delle colpevoli malvagità paterne.

${ }^{20}$ Cfr. Alfieri tragico. 
Il tema ricorre anche in Manasse, altra tragedia del padre Granelli, il quale divenne re di Giuda (699-639 a.C.) succedendo appena dodicenne al padre Ezechia. A lui la tradizione vetero-testamentaria assegna la responsabilità di una persecuzione da parte dei profeti, episodio ricordato anche nel Saul alfieriano, sebbene portato dall'astigiano al limite più estremo della tragediabilità 21 .

E chiaro quindi che la scelta comune dei drammaturghi gesuiti sul soggetto da rappresentare non fosse casuale: sia nella produzione di Granelli che di Bettinelli la vicenda d'ispirazione biblica è fucina di soggetti dalle caratteristiche ricorrenti; giovani re o figli di re, immediatamente o direttamente coinvolti nella gestione del potere, rivolti al raggiungimento dello stesso o al suo mantenimento. Il tragico cristiano di queste opere appare perciò inevitabilmente compromesso con la sfera della politica (o della Ragion di Stato), chiaramente diretto ad un'edificazione e a un miglioramento dell'animus degli educandi non soltanto in ambito spirituale ma soprattutto per ciò che concerne il potere e l'amministrazione di uno Stato, compiti cioè che i rampolli delle famiglie più importanti avrebbero con ogni probabilità assunto su di sé nel corso della vita.

In questo senso deve anche essere giustificata la forte virata in favore della storia 'laica' di Bettinelli che dall'esordio del Gionata passa al Demetrio Poliorcete e quindi al Serse re di Persia; ho mostrato altrove che la scelta non fu affatto casuale, ma determinata direttamente dalla vita politica (e anche culturale) di Filippo di Borbone nel ducato di Parma e dal suo ministro Guillame du Tillot ${ }^{22}$. In questa attenzione alla realtà politica, al mondo contemporaneo, nell'anelito a una maggiore umanità e umanizzazione del potere si concretizza "un tragico moderno e tutto gesuitico, che mira alla 'correzion del costume' e, al di là del semplice didascalismo, crede nell'esemplarità della storia sacra" 23 . E in tale esemplarità - io aggiungerei 'politica' - della storia sacra aveva confidato anche Alfieri per il più famoso Saul prima (1783) e per l'Abele (postumo 1790), ma anche Alfonso Varano nel Giovanni di Giscala ${ }^{24}$ o Giovan Battista Roberti per l'Adonia25,

\footnotetext{
${ }^{21}$ Cfr. Masiello, Lideologia tragica di Vittorio Alfieri.

${ }^{22}$ Cfr. Tiranni a teatro.

23 Zanlonghi, Teatri di formazione, p. 307; si veda inoltre Zanlonghi, "Il teatro nella pedagogia gesuitica", pp. 159-190.

${ }^{24}$ Cfr. Varano, Giovanni di Giscala.
}

${ }^{25}$ Cfr. Sandonà, Ragione e carità. L'opera è del 1757, ed in essa si descrive l'ambizione di Adonia, primogenito della casa di David, di essere unto re mentre Dio aveva predestinato Salomone, il figlio di Betsabea; il pentito ravvedimento di 
o lo stesso Pietro Metastasio che nel 1732 compose La morte di Abel. Dunque il tema era assai diffuso e direi anche in misura sorprendente se solo si considera che siamo in piena età illuministica e ci si spinge addirittura appena a ridosso delle sollevazioni rivoluzionarie francesi. Ma questo tema, per contro, assume nelle singole menti sfaccettature e inclinazioni differenti eppure riconducibili ad un bisogno primario comune.

Il tragico cristiano ${ }^{26}$ adottato da Alfieri per il Saul poteva certamente contare su quella "venerabilità preventiva" che egli stesso denunciava come imprescindibile tramite per la 'vera tragedia', giudicando la modernità impossibilitata ontologicamente a produrre e quindi rappresentare eroi compiutamente tragici. Così in Abele l'attenzione del tragico è rivolta a colpire e suscitare i sensi (non solo l'intelletto) del pubblico e in Saul la ragionevolezza del re è offuscata dalla soverchieria (predominante anche a costo della vita) del potere. Ma laddove il conflitto delle passioni del primo Alfieri ${ }^{27}$ e in generale della drammaturgia gesuitica si era incentrato su una rappresentazione del potere chiusa nel conflitto contenuto nell'idea stessa di potere e cioè nello scontro tra lo Stato e gli affetti familiari, tra la legge politica e quella affettiva, il Saul appare slegarsi da ogni vincolo di natura terrena, per spostare ancora più su il livello dello scontro. Laddove, cioè, la drammaturgia gesuitica si era incentrata in particolare su una corretta gestione del potere (naturalmente ispirata da contenuti morali della religione cristiana attraverso la mediazione della magnanimità della drammaturgia classica seicentesca) giungendo alla scelta della storia laica dopo un percorso letterario-ideologico pienamente cristiano, Alfieri invece (con un processo tematico esattamente inverso) scardina dall'interno l'idea stessa del potere, depurandolo da qualsivoglia contatto terreno; il Saul alfieriano appare allora conforme solo esteriormente alla trattazione 'politica' dei gesuiti, e la tematica biblica rappresenta un mero tramite contenutistico per un progetto ideologico ben più elevato. Ciò rappresenta piuttosto l'estrema assolutizzazione del conflitto intrinseco al potere stesso: Alfieri sposta l'oggetto della trattazione dal piano squisitamente politico dell'amministrazione terrena a quello ideologico della sacralità regale di Saul, impegnato a difendere la sua condizione di re non da oscuri nemici interni alla corte, ministri machiavellici o nemici più potenti; l'intento di Saul è

Adonia per la paura dell'ira divina mette fine alla saga che si conclude con il riconoscimento di Salomone come legittimo re.

${ }^{26}$ Cfr. Barberi Squarotti, Il tragico cristiano; si veda, inoltre, Ghidetti in Alfieri tragico, pp. 637-655.

${ }^{27}$ Cfr. Santato, Tra mito e palinodia; Barsotti, Alfieri e la scena. 
quello di conservare in eterno la sua regalità, sfidando nell'estremo duello Dio stesso e individuando nel suicidio il tramite per l'eternazione della sua condizione di re. Laddove il teatro gesuitico e la precedente produzione alfieriana si erano attestati su una dimensione tutta terrena della questiona politica, con il Saul tutto è assolutizzato: il concetto stesso di potere-regalità, la sfida, la punizione la soluzione finale: "Empia Filiste, / me troverai, ma almen da re, qui ... morto." Un re che muore sulla sua stessa spada (volutamente modificando in questo punto la narrazione biblica che invece descrive la decapitazione come opera dei Filistei, Samuele I, 4-10), è un re non vinto, la cui corona rotola nella polvere e nel sangue ancora e per sempre sul capo di Saul; la morte del re suicida è, dunque, il tramite per l'assoluto: Saul sarà per sempre re e non il profeta Samuele, non David e nemmeno Dio potranno mai privarlo della sua regalità. Il contesto politico che premeva con la sua urgenza sulla produzione teatrale dei gesuiti perde i tratti della immediata contingenza nel Saul di Alfieri che, invece, vi aveva dedicato le 'tragedie della libertà' e la produzione trattatistica; resta tuttavia nella tragedia più luminosa dell'astigiano la consapevolezza dell'ingombrante e irremovibile presenza del mondo politico nella vita dell'uomo come nella riflessione del letterato, aprendo definitivamente il campo alla drammaturgia borghese e risorgimentale dell'Ottocento e riaffermando con 'aristocratico distacco', proprio attraverso la mediazione tematica delle sacre scritture, l'imprescindibilità dell'antico principio umanistico degli intellettuali guida e lume - è il caso di dirlo — per il potere.

\section{Università di Bari}

\section{Opere citate}

Alfieri tragico, a cura di Enrico Ghidetti e Roberta Turchi. Firenze: Le Lettere, 2003 (numero speciale de La Rassegna della Letteratura Italiana, 107:2 (lugliodicembre 2003).

Alfieri, Vittorio. Vita, a cura di Giampaolo Dossena. Torino: Einaudi, 1974.

Alfieri, Vittorio, Parere sulle tragedie e altre prose critiche, a cura di Morena Pagliai. Asti: Casa d'Alferi, 1978.

Ariani, Marco. "Lossessione delle 'regole' e il disordine degli 'affetti': lineamenti di una teoria illuministica del teatro tragico" in Il teatro italiano del Settecento, a cura di Gerardo Guccini. Bologna: Il Mulino, 1988, pp. 121-148.

Bancheri, Salvatore. "Polemiche sulla tragedia sacra regolare fra Sei e Settecento" in Romance Languages Annual, 2 (1990): 177-181.

Barberi Squarotti, Giorgio. Il tragico cristiano. Da Dante ai moderni. Firenze: Leo S. Olschki, 2003.

Barsotti, Anna. Alfieri e la scena. Da fantasmi di personaggi a fantasmi di spettatori. Roma: Bulzoni, 2001. 
Bertana, Emilio. La tragedia. "Storia dei generi letterari italiani." Milano: Vallardi, 1905.

Bertana, Emilio. "Il teatro tragico italiano del secolo XVIII prima dell'Alfieri" numero monografico di Giornale storico della letteratura italiana, suppl. 4 (1901).

Bettinelli, Saverio. "Discorso sopra il teatro italiano" in Illuministi italiani. Opere di Francesco Algarotti e Saverio Bettinelli, a cura di Ettore Bonora. MilanoNapoli: Ricciardi, 1969.

Bettinelli, Saverio. Tomo sesto che contiene tragedie e poesie varie, Zatta, Venezia 1782.

Binni, Walter. "L'aspirazione al teatro tragico", in Storia della letteratura italiana, diretta da Emilio Cecchi-Natalino Sapegno, vol. 6 (Milano: Garzanti, 1968), pp. 415-426.

Bonora, Ettore. "Le tragedie e la poetica del tragico di Saverio Bettinelli" in Parini e altro Settecento, Milano: Feltrinelli, 1982, pp. 156-179.

Brizzi, Gian Paolo. "Caratteri ed evoluzione del teatro di collegio italiano" in Cattolicesimo e lumi nel Settecento italiano, a cura di Mario Rosa. Roma: Herder, 1981, pp. 177-204.

Carli, Gian Rinaldo. Dell'indole del teatro tragico antico e moderno (1747), in Opere. Milano: Nell'Imperiale Monastero di Sant'Ambrogio Maggiore, 1787, tomo XVII, pp. 1-191.

Carlson, Marvin Lee. Teorie del teatro. Bologna: Il Mulino, 1996.

Concina, Daniele. De spectaculis theatralibus dissertationes duae. Roma: Barbiellini, 1752.

Concina, Daniele. Dé teatri moderni contrari alla professione cristiana libri due. Roma: Barbiellini, 1755.

De Angelis, V. Critiche, traduzioni ed imitazioni del teatro di G. Racine durante il secolo XVIII. Arpino: Fraioli, 1914.

De Carli, Antonio Riflessi francesi nell'opera di Saverio Bettinelli. Torino: Chiantore, 1928.

Doglio, Federico. Il teatro tragico italiano. Storia e testi. Parma: Guanda, 1960.

Ferrari, Luigi. Le traduzioni italiane del teatro tragico francese nei secoli XVII e XVIII. Parigi: Champion, 1925.

Ferrari, Luigi. "Appunti sul teatro tragico dei Gesuiti in Italia" in Rassegna bibliografica della letteratura italiana, 7 (1899): 124-130.

Ferrone, Siro eTeresa Megale, Il teatro, in Storia della letteratura italiana, diretta da Enrico Malato, vol. VI. Il Settecento. Roma: Salerno, 1998.

La festa a Roma dal Rinascimento al 1870, a cura di Marcello Fagiolo. Roma: Allemandi, 1997.

Galletti Alfredo, Le teorie drammatiche e la tragedia nel secolo XVIII. Cremona: Fezzi, 1901.

I Gesuiti e $i$ primordi del teatro barocco in Europa, Atti del Convegno di studi (Roma-Anagni 26-30 ottobre 1994), a cura di Maria Chiabò e Federico Doglio. Roma: La Torre d'Orfeo, 1995.

I Gesuiti e la Ratio Studionum, a cura di Manfred Hinz, Roberto Righi, Danilo 
Zardin. Roma: Bulzoni, 2004.

Granelli, Giovanni. Seila figlia di Jefte, Modena: Soliani, 1765.

Granelli, Giovanni. Manasse Re Di Giuda. Sedecia Ultimo Re Di Giuda. Dione

Siracusano, Bologna: per Girolamo Corciolani, ed Eredi Colli a S. Tommaso d'Aquino, 1751-1754.

Herr, Mireille. Les tragédies bibliques au XVIII siecle, Parigi-Ginevra: ChampionSlatkine, 1988.

Luciani, Paola. Le passioni e gli affetti. Studi sul teatro tragico del Settecento. Pisa: Pacini, 1999.

Martello, Pier Jacopo. Della tragedia antica e moderna (1714), in Scritti critici e satirici, a cura di Hannibal S. Noce, Laterza, Bari 1963, pp. 187-316.

Mattioda, Enrico. Le teorie della tragedia nel Settecento. Modena: Mucchi, 1994.

Mangini, Nicola. "Il teatro tragico francese in Italia nel secolo XVIII" in Drammaturgia e spettacolo tra Sette e Ottocento. Studi e ricerche. Padova: Liana Editrice, 1979, pp. 1-20.

Marotti, Ferruccio. Lo spazio scenico. Teorie e tecniche scenografiche in Italia dall'età barocca al Settecento. Roma: Bulzoni, 1974.

Masiello, Vitilio Lideologia tragica di Vittorio Alfieri. Roma: Edizioni dell'Ateneo, 1964.

Meregazzi, G. Le tragedie di Pierre Corneille nelle traduzioni e imitazioni italiane del secolo XVIII. Bergamo: Fagnani: 1906.

Minervini, Francesco S. "L'etica aristotelica e la progettualità gesuitica del teatro di Corneille e Bettinelli" in Annali della Facoltà di Lettere e Filosofia dell'Università di Bari, 43 (2000), pp. 51-75.

Muratori, Ludovico Antonio. Della perfetta poesia italiana, a cura di Ada Ruschioni, Marzorati, Milano 1971.

Orfeo in Arcadia. Studi sul teatro a Roma nel Settecento, a cura di Giorgio Petrocchi. Roma: Istituto della Enciclopedia Italiana, 1984.

Puppo, Mario. Il Romanticismo. Roma: Studium, 1951.

Ragion di Stato a Teatro, Atti del Convegno (Foggia, Lucera, Bari 18-20 aprile 2002), a cura di Stella Castellaneta e Francesco S. Minervini. Taranto: Lisi, 2005.

Regulae Rectoris n. 13, Ratio Studiorum et Institutiones Scholasticae Societatis Jesu, a cura di Michel Pachtler. Tomo 2. Berlino: Hofmann, 1887.

Sandonà, Giovan Battista. Ragione e carità. Per un ritratto di Giambattista Roberti. Venezia Istituto Veneto di scienze, lettere ed arti, 2002.

Sannia Nowé, Laura. "Epifanie e metamorfosi della clemenza nella letteratura drammaturgica del Settecento" in La cultura fra Sei e Settecento, a cura di Elena Sala De Felice e Laura Sannia Nowé. Modena: Mucchi, 1994, pp. 171-196.

Santato, Guido. Tra mito e palinodia. Itinerari alfierani. Modena: Mucchi, 1999.

Scaduto, Mario. "Il teatro gesuitico" in Archivium Historicum Soc. Iesu, 36 (1967): 194-215.

Simioni, Attilio. Per la storia del teatro gesuitico in Italia nel XVIII secolo. Napoli: Jovene, 1907. 
Tiranni a teatro. Demetrio Poliorcete e Serse re di Persia di Saverio Bettinelli, a cura di Francesco S. Minervini. Bari: Palomar, 2002.

Varano, Alfonso. Giovanni di Giscala, in Tragedie del Settecento, a cura di Enrico Mattioda, Mucchi, Modena 1999, vol. 2, pp. 9-130

Zanlonghi, Giovanna. Teatri di formazione: actio, parola e immagine nella scena gesuitica del Sei-Settecento a Milano. Milano: Vita e Pensiero, 2002.

Zanlonghi, Giovanna "Il teatro nella pedagogia gesuitica: una 'scuola di virtù'" in I Gesuiti e la Ratio Studiorum, a cura di Manfred Hinz, Roberto Righi, Danilo Zardin. Roma: Bulzoni, 2004, pp. 159-190. 